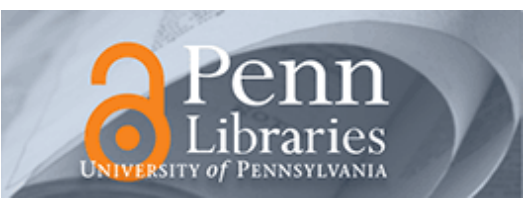

University of Pennsylvania

ScholarlyCommons

\title{
Ultra-Thin Super High Frequency Two-Port ALN Contour-Mode Resonators and Filters
}

\author{
Matteo Rinaldi \\ University of Pennsylvania, rinaldim@seas.upenn.edu \\ Chiara Zuniga \\ University of Pennsylvania, zunigac@seas.upenn.edu \\ Chnegjie Zuo \\ University of Pennsylvania, chengjiezuo@hotmail.com
}

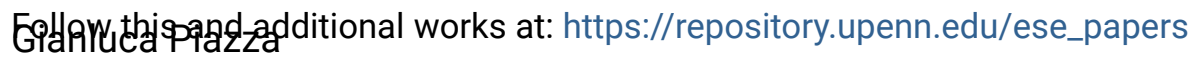

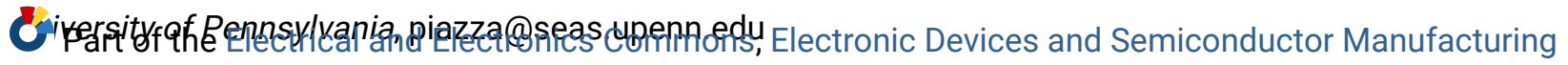

Commons, Nanoscience and Nanotechnology Commons, and the Nanotechnology Fabrication Commons

\section{Recommended Citation}

Matteo Rinaldi, Chiara Zuniga, Chnegjie Zuo, and Gianluca Piazza, "Ultra-Thin Super High Frequency TwoPort ALN Contour-Mode Resonators and Filters", . June 2009.

Copyright 2009 IEEE. Reprinted from:

Rinaldi, M.; Zuniga, C.; Zuo, C.; Piazza, G., "Ultra-thin Super High Frequency two-port ALN contour-mode resonators and filters," Solid-State Sensors, Actuators and Microsystems Conference, 2009. TRANSDUCERS 2009.

International , vol., no., pp.577-580, 21-25 June 2009

Publisher URL: http://ieeexplore.iee.org/stamp/stamp.jsp?arnumber=5285390\&isnumber $=5285368$

Digital Object Identifier: 10.1109/SENSOR.2009.5285390

This material is posted here with permission of the IEEE. Such permission of the IEEE does not in any way imply IEEE endorsement of any of the University of Pennsylvania's products or services. Internal or personal use of this material is permitted. However, permission to reprint/republish this material for advertising or promotional purposes or for creating new collective works for resale or redistribution must be obtained from the IEEE by writing to pubs-permissions@ieee.org. By choosing to view this document, you agree to all provisions of the copyright laws protecting it.

This paper is posted at ScholarlyCommons. https://repository.upenn.edu/ese_papers/523

For more information, please contact repository@pobox.upenn.edu. 


\title{
Ultra-Thin Super High Frequency Two-Port ALN Contour-Mode Resonators and Filters
}

\begin{abstract}
This paper reports on the demonstration of a new class of ultra-thin ( $250 \mathrm{~nm}$ thick) super high frequency (SHF) AIN piezoelectric two-port resonators and filters. A thickness field excitation scheme was employed to excite a higher order contour extensional mode of vibration in an AIN nano plate ( $250 \mathrm{~nm}$ thick) above $3 \mathrm{GHz}$ and synthesize a $1.96 \mathrm{GHz}$ narrow-bandwidth channel-select filter. The devices of this work are able to operate over a frequency range from 1.9 to $3.5 \mathrm{GHz}$ and are employed to synthesize the highest frequency MEMS filter based on electrically self-coupled AIN contour-mode resonators. Very narrow bandwidth $(\sim 0.35 \%)$ and high off-band rejection $(\sim 35 \mathrm{~dB})$ were achieved at an operating frequency of $1.96 \mathrm{GHz}$. This first prototype showed insertion loss of $11 \mathrm{~dB}$, which can be improved to few $\mathrm{dB}$ if parasitic elements are eliminated or device capacitance is increased.
\end{abstract}

\section{Keywords}

Contour-Mode Resonators, MEMS Resonators, MEMS Filters, NEMS

\section{Disciplines}

Electrical and Electronics | Electronic Devices and Semiconductor Manufacturing | Nanoscience and Nanotechnology | Nanotechnology Fabrication

\section{Comments}

Copyright 2009 IEEE. Reprinted from:

Rinaldi, M.; Zuniga, C.; Zuo, C.; Piazza, G., "Ultra-thin Super High Frequency two-port ALN contour-mode resonators and filters," Solid-State Sensors, Actuators and Microsystems Conference, 2009.

TRANSDUCERS 2009. International , vol., no., pp.577-580, 21-25 June 2009

Publisher URL: http://ieeexplore.ieee.org/stamp/stamp.jsp?arnumber=5285390\&isnumber=5285368

Digital Object Identifier: 10.1109/SENSOR.2009.5285390

This material is posted here with permission of the IEEE. Such permission of the IEEE does not in any way imply IEEE endorsement of any of the University of Pennsylvania's products or services. Internal or personal use of this material is permitted. However, permission to reprint/republish this material for advertising or promotional purposes or for creating new collective works for resale or redistribution must be obtained from the IEEE by writing to pubs-permissions@ieee.org. By choosing to view this document, you agree to all provisions of the copyright laws protecting it. 


\title{
ULTRA-THIN SUPER HIGH FREQUENCY TWO-PORT ALN CONTOUR-MODE RESONATORS AND FILTERS
}

\author{
M. Rinaldi ${ }^{*}$, C. Zuniga, C. Zuo and G. Piazza \\ University of Pennsylvania, Philadelphia, Pennsylvania, USA
}

\begin{abstract}
This paper reports on the demonstration of a new class of ultra-thin (250 nm thick) Super High Frequency (SHF) AlN piezoelectric two-port resonators and filters. A thickness field excitation scheme was employed to excite a higher order contour extensional mode of vibration in an AlN nano plate (250 $\mathrm{nm}$ thick) above $3 \mathrm{GHz}$ and synthesize a $1.96 \mathrm{GHz}$ narrow-bandwidth channel-select filter. The devices of this work are able to operate over a frequency range from 1.9 to $3.5 \mathrm{GHz}$ and are employed to synthesize the highest frequency MEMS filter based on electrically self-coupled AlN contour-mode resonators. Very narrow bandwidth $(\sim 0.35 \%)$ and high off-band rejection $(\sim 35 \mathrm{~dB})$ were achieved at an operating frequency of $1.96 \mathrm{GHz}$. This first prototype showed insertion loss of $11 \mathrm{~dB}$, which can be improved to few $\mathrm{dB}$ if parasitic elements are eliminated or device capacitance is increased.
\end{abstract}

\section{KEYWORDS}

Resonators, MEMS filters, contour-mode resonators, aluminum nitride (AlN) film, Super High Frequency.

\section{INTRODUCTION}

In recent years the demand for single-chip multi-band RF solutions for advanced wireless communication systems has been growing steadily. This dictates the need for compact, low cost and high performance RF components. A very promising solution to synthesize integrated and high-performance RF filters is based on microelectromechanical (MEMS) resonators. Different resonator technologies based on electrostatic [1,2] or piezoelectric [3] transduction have been investigated and among these, Aluminum Nitride (AlN) Contour-Mode MEMS resonator (CMR) [3] has emerged as one of the most promising in enabling the fabrication of multiple frequency and high performance resonators on the same silicon chip [3,4]. This very important feature makes the CMR technology preferable to the conventional FBAR or quartz crystal technologies, for which only one frequency of operation can be achieved on a single silicon substrate. In addition, the reduced dimension of CMR devices and their potential integration with CMOS favor their employment, instead of cumbersome and unintegrable SAW devices, for the synthesis of next generation singlechip RF front-ends.

Nevertheless, the frequency of operation of CMR devices has been limited to the UHF range and the capability of this technology to provide for SHF RF devices has not been explored to date. Operation in the SHF will open new opportunities for this technology for the demonstration of RF channel selectors for radar applications or their use in emerging wireless standards such as WiFi. SHF one-port NEMS laterally-vibrating AlN resonators based on lateral field excitation (LFE) have been recently demonstrated by our group [5], but the absence of the bottom electrode (not necessary for LFE) prevented us from easily configuring the nano resonators as two-port networks, and hence from fabricating narrow band filters by cascading multiple stages.

In this work, a thickness field excitation scheme (TFE) was employed to excite a higher order contour extensional mode of vibration in an AlN nano plate ( $250 \mathrm{~nm}$ thick) above $3 \mathrm{GHz}$. Such a thin film is essential to guarantee operations at SHF with low motional resistance (180$440 \Omega$ ) and a very compact form factor. In fact scaling the film thickness to few hundred nanometers raises the device capacitance above the high frequency substrate parasitics, and reduces its footprint to a small area of approximately $17 \times 50 \mu \mathrm{m}$. The thickness field excitation enables the use of the resonator as a two port device and attains values of the electromechanical coupling, $k_{t}^{2}$, of approximately $1.8 \%$. This value is at least 5 times higher than what previously demonstrated $(\sim 0.3 \%)$ by the same group for SHF LFE resonators [5].

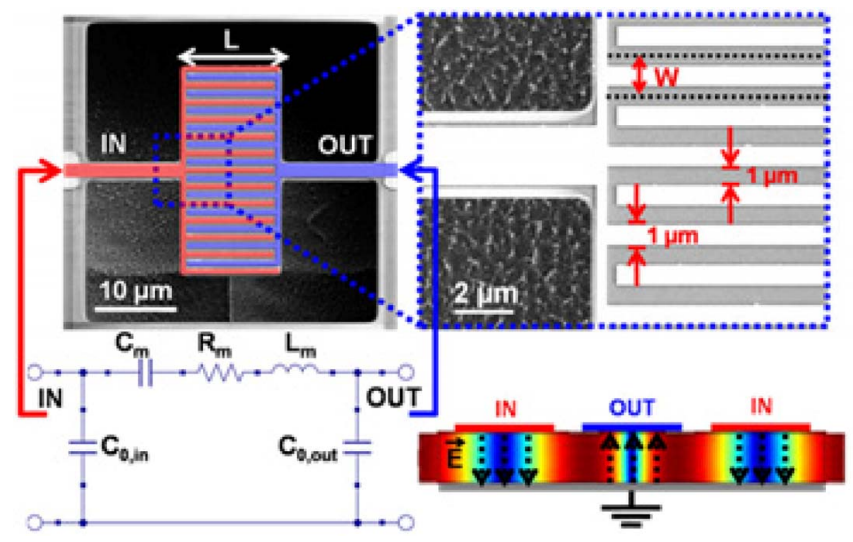

Figure 1: SEM picture and equivalent electrical circuit (BVD) of a TFE $1.9 \mathrm{GHz}$ AlN two-port CMR. The FEM simulation represents the mode of vibration of the resonator.

Two port devices operating between 1.9 and $3.5 \mathrm{GHz}$ were demonstrated and the highest frequency $(1.96 \mathrm{GHz})$ MEMS filter based on self-coupled AlN controur-mode resonators [6] was synthesized by cascading two two-port 
resonators. Narrow bandwidth $(\sim 0.35 \%)$ and high offband rejection $(\sim 35 \mathrm{~dB})$ were demonstrated. This first prototype showed insertion loss of $11 \mathrm{~dB}$, which can be significantly improved if parasitic elements are eliminated or device capacitance is further increased.

\section{DESIGN}

High performance two-port AlN CMRs in the Very High Frequency (VHF) range with quality factor between 1,000 and 2,000 have been previously demonstrated $[3,4]$. In this work, the device layout has been modified to overcome the fundamental transduction challenges associated with previously unexplored frequencies in the SHF range. The device dimensions have been scaled both in the lateral and vertical directions and an unconventional aspect ratio $(n W>L$, Fig. 1) for the CMR has been introduced in order to achieve higher operating frequencies along with low values of motional impedance.

\section{SHF Two-port AIN Contour-Mode Resonator}

The SHF AlN resonator (Fig. 1) consists of a $250 \mathrm{~nm}$ thick AlN film sandwiched between a bottom Pt $(50 \mathrm{~nm})$ and a top $\mathrm{Au}(100 \mathrm{~nm})$ electrodes. The bottom Pt is a single electrode connected to electrical ground, while the top $\mathrm{Au}$ layer (chosen for its very low resistivity) is patterned in $n$ parallel electrodes whose width varies between 0.5 and $1 \mu \mathrm{m}$ depending upon the desired frequency of operation.

A number $n_{\text {in }}$ of the top Au electrodes are connected to form the input port and the remaining $\left(n_{\text {out }}\right)$ are used to form the output port. When an AC signal is applied to the input port, the electric field across the thickness of the AIN causes an in plane deformation of the structure through the equivalent $d_{31}$ piezoelectric coefficient and excites the resonator in a contour-extensional mode of vibration (TFE excitation scheme). Because of the direct piezoelectric effect, charge is generated and collected by the sensing electrodes (output port). The entire structure of Figure 1 can be seen as a number, $n_{i n}$, of mechanically coupled subresonators (of width $W$ ) forming the input port and a number, $n_{\text {out }}$, of them forming the output port. The resonance frequency of the whole structure can be lithographically set as expressed in equation (1):

$$
f_{0}=\frac{1}{2 W} \sqrt{\frac{E_{p}}{\rho}}
$$

where $W$ is the width of each sub-resonator and $E_{p}$ and $\rho$ are respectively the equivalent Young's modulus and density of AlN.

As reported in [7] the described mechanical microstructure can be modeled through an equivalent Butterworth van Dyke (BVD) circuit (Fig. 1) where each electrical element can be expressed in terms of the geometrical dimensions and the material properties of the micromechanical structure itself (2):

$$
\begin{gathered}
C_{0, \text { in }}=n_{\text {in }} \varepsilon_{0} \varepsilon_{33} \frac{W L}{T} \quad C_{0, \text { out }}=n_{\text {out }} \varepsilon_{0} \varepsilon_{33} \frac{W L}{T} \\
R_{m}=\frac{n_{\text {in }}+n_{\text {out }}}{n_{\text {in }} n_{\text {out }}} \frac{\pi}{8} \frac{T}{L} \frac{\rho^{1 / 2}}{d_{31}^{2} E_{p}^{3 / 2} Q} \\
C_{m}=\frac{n_{\text {in }} n_{\text {out }}}{n_{\text {in }}+n_{\text {out }}} \frac{8}{\pi^{2}} \frac{W L}{T} E_{p} d_{31}^{2} \\
L_{m}=\frac{n_{\text {in }}+n_{\text {out }}}{n_{\text {in }} n_{\text {out }}} \frac{1}{8} \frac{T W}{L} \frac{\rho}{E_{p}^{2} d_{31}^{2}}
\end{gathered}
$$

where $\varepsilon_{0}$ is the free space permittivity, $\varepsilon_{33}$ the AlN permittivity; $W, L$ and $T$ are the width, length and thickness of each sub-resonator and $Q$ is the resonator quality factor.

According to (1), the width, $W$, of each sub-resonator has to be reduced in order to increase the device resonance frequency into the SHF band. Nonetheless, (2) suggests that a reduction of $W$ needs to be compensated by an increase in the $n L / T$ ratio in order to keep the device impedance small and above the high frequency parasitics. It is important to note that an excessive increase in the device length $(L)$ along with the scaling of its width $(W)$ would cause an intolerable increase of the electrical resistance of the electrodes and consequently load the $Q$ of the resonator. Therefore, the scaling of CMR devices to frequencies above few $\mathrm{GHz}$ requires that the thickness of the AlN layer is reduced below $1 \mu \mathrm{m}$. On the other hand, the degradation of the piezoelectric properties and film quality in thin AlN layers, due to misoriented grains at the electrode/piezoelectric interface region [8], needs to be taken into account.

In this perspective, the thickness of the AlN film was scaled to $250 \mathrm{~nm}$ in order to design and fabricate CMR devices operating at frequencies well above $3 \mathrm{GHz}$. Furthermore, in collaboration with Tegal Corporation, the quality of sputtered deposited AlN thin films on Pt seed layer (bottom electrode) was optimized and attained rocking curve values as good as $2.1^{\circ}$ (equivalent to what has been obtained in micron-size devices). The width, $W$, of each sub-resonator was varied between 2 and $1 \mu \mathrm{m}$, to provide frequencies of operation between 1.9 and $3.5 \mathrm{GHz}$. The device length, $L$, was set to $17 \mu \mathrm{m}$. Additionally, in order to further reduce the resonator motional resistance the number $n_{\text {in }}$ and $n_{\text {out }}$ of sub-resonators at the input and output ports was varied between 12 and 24 . This solution gives an unconventional aspect ratio $(n W>L)$ for the overall AlN plate that forms the CMR device. Despite this, device operation without spurious responses was preserved.

\subsection{GHz Narrow-band AIN CMR Filter}

The use of TFE permits to configure the CMR device as a two-port network to readily synthesize filters and 
yields a value of the electromechanical coupling, $k_{t}^{2}$, at least $5 \mathrm{X}$ the ones previously reported [5] for SHF lateral field excited NEMS CMRs. Thanks to this, two TFE devices were cascaded in series to form a narrow band filter operating at $1.96 \mathrm{GHz}$.

The coupling technique is based on the use of the intrinsic capacitance of a two-port device and permits the definition of band pass filters by simply employing same frequency resonators [6]. This is different from conventional ladder configurations [9], which require two different frequency devices with an associated impact on filter yields.

The equivalent electrical model of the two-stage AlN CMR filter is shown in Figure 2. Since filter bandwidth, $B W_{3 d B}$, and insertion loss, $I L$, are directly related to the device $k_{t}^{2}-Q$ product, it is convenient to explicitly express the dependence of the equivalent electrical components in (2) on $k_{t}^{2}-Q(3)$.

$$
\begin{gathered}
C_{0}=C_{0, \text { in }}+C_{0, \text { out }}=\left(n_{\text {in }}+n_{\text {out }}\right) \varepsilon_{0} \varepsilon_{33} \frac{W L}{T} \\
R_{m}=\frac{\pi^{2}}{2} \frac{1}{\omega_{0} C_{0} k_{t}^{2} Q} ; \quad C_{m}=\frac{2}{\pi^{2}} C_{0} k_{t}^{2} \\
L_{m}=\frac{\pi^{2}}{2} \frac{1}{\omega_{0}^{2} C_{0} k_{t}^{2}} ; \quad \omega_{0}=2 \pi f_{0}
\end{gathered}
$$

Neglecting the parasitic components and assuming values of $k_{t}{ }^{2}-Q>>1$, the IL of the system can be expressed as in (4) and its bandwidth as in (5).

$$
\begin{gathered}
I L \approx-20 \log _{10}\left[\frac{2}{2+\pi^{2} / k_{t}^{2} Q}\right] \\
B W_{3 d B} \approx \sqrt{1+\frac{2 C_{m}}{C_{0}}}-1 \approx \frac{C_{m}}{C_{0}}
\end{gathered}
$$

It is important to note that the presence of parasitic components $\left(C_{p} \approx C_{p 1}+C_{p 2} / 2\right.$ and $R_{p}$, Fig. 2) reduces the effective $k_{t}^{2}$ of the filter and limits its value according to (6).

$$
k_{t, \text { eff }}^{2} \approx \frac{\pi^{2}}{2} \frac{C_{m}}{C_{0}+C_{p}}
$$

It is therefore clear that it is extremely important to maintain values of the device capacitance, $C_{0}$, well above the parasitics when scaling the device to higher frequencies of operation. This explains the need for ultrathin AlN films, which enable higher values of capacitance, $C_{0}$, for a given device footprint.

\section{EXPERIMENTAL RESULTS}

The devices were fabricated combining optical and electron-beam lithography techniques. A 4 mask postCMOS compatible fabrication process analogue to the one presented in [6] was employed.
The fabricated devices were tested in an RF probe station and the scattering parameters were measured by an Agilent ${ }^{\circledR}$ N5230A Network Analyzer after performing a short-open-load-through (SOLT) calibration on a reference substrate.

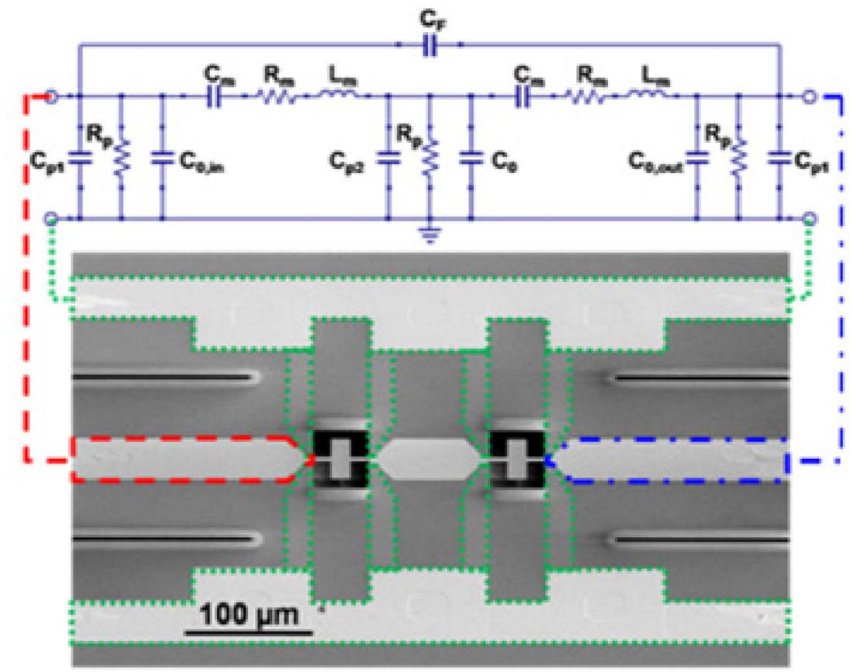

Figure 2: SEM picture and equivalent electrical circuit of the $1.96 \mathrm{GHz} 2^{\text {nd }}$ order filter based on electrically self-coupled AlN CMRs.

The characteristic response of a $3.46 \mathrm{GHz}$ two-port CMR is shown in Figure 3.

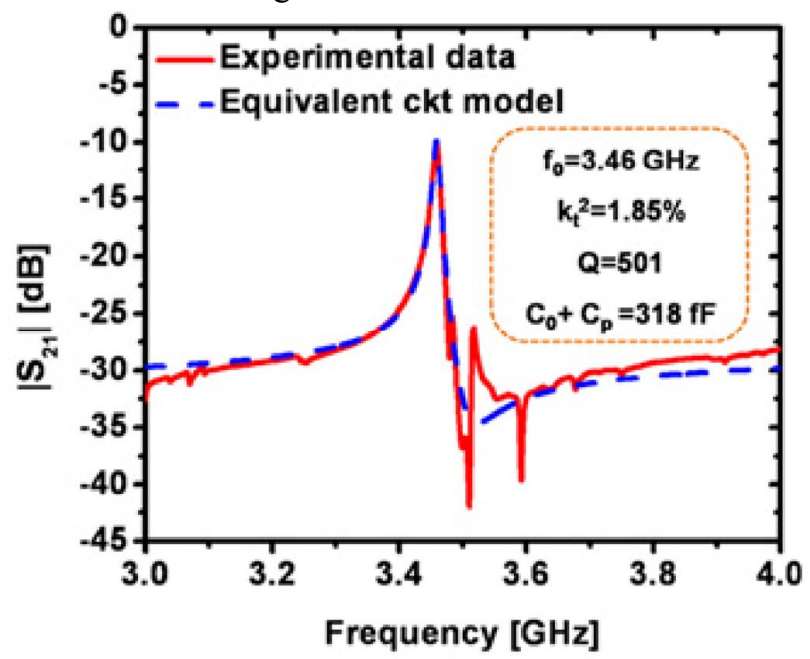

Figure 3: Measured electrical response and equivalent circuit model fitting of a fabricated $3.46 \mathrm{GHz}$ AlN two-port CMR.

The measured data were fitted to the circuit model shown in Figure 2 and the extracted equivalent parameters for this device and others operating at lower frequencies are reported in Table 1. The data confirms that values of intrinsic electromechanical coupling, $k_{t}^{2}$, as high as $1.85 \%$ were attained for the fabricated two-port TFE devices.

Two of these two-port resonators were electrically cascaded to form a $2^{\text {nd }}$ order filter operating at $1.96 \mathrm{GHz}$. The measured transmission $\left(S_{21}\right)$ of this device is shown in 
Figure 4. The response of the filter was fitted to the previously described circuit model (Fig. 2). The extracted values of $k_{t}^{2}$ and $Q$ along with the device performances are reported in Figure 4.

Table 1: Electromechanical parameters extracted from the measured S parameters of the fabricated two-port AlN CMRs.

\begin{tabular}{|c|c|c|c|c|}
\hline $\begin{array}{c}\text { Frequency of } \\
\text { Operation }\end{array}$ & $\boldsymbol{k}_{\boldsymbol{t}}{ }^{2}$ & $\boldsymbol{Q}$ & $\boldsymbol{R}_{\boldsymbol{M}}$ & $\boldsymbol{C}_{\boldsymbol{0}}+\boldsymbol{C}_{\boldsymbol{p}}$ \\
\hline $\mathbf{1 . 8 7} \mathbf{G H z}$ & $1.84 \%$ & 387 & $440 \Omega$ & $303 \mathrm{fF}$ \\
\hline $\mathbf{3 . 0 4} \mathbf{~ G H z}$ & $1.5 \%$ & 517 & $225 \Omega$ & $301 \mathrm{fF}$ \\
\hline $\mathbf{3 . 4 6} \mathbf{G H z}$ & $1.85 \%$ & 501 & $180 \Omega$ & $318 \mathrm{fF}$ \\
\hline
\end{tabular}



Figure 4: Measured electrical response and equivalent circuit model fitting (before and after de-embedding) of a fabricated $1.96 \mathrm{GHz} 2^{\text {nd }}$ order filter based on electrically self-coupled AlN CMRs. A $500 \Omega$ termination was used for the measurement.

It is important to note that the measured value of $I L$ $(\sim 11 \mathrm{~dB})$ is significantly affected by the parasitic components $C_{p}$ and $R_{p}$. In fact, as previously explained, the presence of parasitics reduces the effective value of the electromechanical coupling, $k_{t, \text { eff, }}{ }^{2}$ to approximately half $(1.05 \%)$ of the one intrinsic to the device $(1.84 \%)$. In order to simulate the de-embedded response of the filter (Fig. 4), the parasitic capacitance, $C_{p l}$, was measured by means of a de-embedding structure fabricated on the same silicon chip, and its value was subtracted to the fitted total input capacitance of the filter. After removing all the parasitic components from the equivalent circuit of the filter, the de-embedded response (Fig. 4) shows a reduced value of $I L$ of approximately $5.8 \mathrm{~dB}$. This same value of $I L$ could be alternatively attained by making the device capacitance, $C_{0}$, larger and dominant over the parasitic components. Additionally, the parasitics can be further reduced by optimizing the configuration of the test pads.

\section{CONCLUSIONS}

Design, fabrication and testing of two-port SHF contour-mode resonators based on thickness field excitation of ultra-thin $(250 \mathrm{~nm})$ AlN films have been demonstrated. Values of electromechanical coupling, $k_{t}^{2}$, comparable to the ones reported for lower frequency devices $(>1.5 \%)$, were achieved at frequencies of operation above $3 \mathrm{GHz}$. The capability of these devices to be employed for the fabrication of narrow band filters has been proven by the realization of the highest frequency $(1.96 \mathrm{GHz}) 2^{\text {nd }}$ order filter based on electrically selfcoupled AlN contour-mode devices. Future work aims at demonstrating even higher frequencies of operation and reducing the filter $I L$ to few $\mathrm{dB}$. In this way, these devices can be effectively employed for radar communications or other emerging wireless standards.

\section{ACKNOLEDGEMENT}

This work was supported by NCMR/NSF grant no. IIS-07-15024, NSF grant no. ECCS-08-22968 and DARPA N/MEMS S\&T grant no. HR-001-06-1-0041. The authors wish to thank the staff of the Wolf Nanofabrication Facility (WNF) at The University of Pennsylvania and Tegal Corporation for their help with the deposition of thin AlN films.

\section{REFERENCES}

[1] K. Wang, A.-C. Wong and C. T.-C. Nguyen, J. Microelectromech. Syst., vol. 9, no. 3, pp. 347-360, 2000.

[2] D. Weinstein, S. A. Bhave, Proceedings IEEE International Electron Devices Meeting '07, pp. 415418, 2007.

[3] G. Piazza, P.J. Stephanou, A.P. Pisano, J. Microelectromech. Syst., vol. 15, no.6, pp. 1406-1418, 2006.

[4] P. J. Stephanou, J. P. Black, A. L. Benjamin, Proceedings IEEE Radio Frequency Integrated Circuits Symposium ‘08, pp. 171-174, 2008.

[5] M. Rinaldi, C. Zuniga, G. Piazza, Proceedings IEEE MEMS'09 Conference, pp. 916-919, 2009.

[6] C. Zuo, et al., Proceedings IEEE Ultrasonics Symposium'07, pp. 1156-1159, 2007.

[7] G. Piazza and A.P. Pisano, Sensors and Actuators A, vol. 136, pp. 638-645, 2007.

[8] J.R. Heffelfinger, D.L. Medlin, and K.F. McCarty, J. Appl. Phys., (85) 1999, 466-472.

[9] G. Piazza, P. J. Stephanou, A. P. Pisano, J. Microelectromech. Syst., vol. 16, no.2, pp. 319-328, 2007.

\section{CONTACT}

* M. Rinaldi, tel: +1-215-573-3276;

rinaldim@seas.upenn.edu. 